\title{
Dual frequency system for power-demanding measurement in the isolated areas
}

\author{
A. Boura, M. Husak \\ Department of Microelectronics, Faculty of Electrical Engineering \\ Czech Technical University in Prague \\ Technicka 2, CZ - 16627 Prague 6, Czech Republic \\ Phone/Fax number: +420224352 237 / +420224310 792, e-mail: bouraa@fel.cvut.cz, husak@fel.cvut.cz
}

\begin{abstract}
The paper presents solution for wireless and battery-less measurement in the enclosed areas. The principle is based on previous work, while this paper is focused on high power-demanding applications such as MEMS accelerometers, gas sensors, piezoresistive strain gauges, etc. It can be suitable for continuous wireless and battery-less measurement in isolated systems such as the rotating objects, concrete walls, enclosed barrels, high temperature chambers etc.

It is based on near magnetic field coupling in radiofrequency band. The principle is similar to the RFID, while it is more powerful and the powering and signal transfer is separated by the different frequencies. The antennas are designed for surface mounting.

The system is desired to be used in long-term monitoring of the environment.
\end{abstract}

\section{Key words}

Wireless powering, Battery-less powering, Wireless sensors, Long-term monitoring, Dual frequency operation

\section{Introduction}

Measurement in the isolated systems is crucial task in many areas of interest. Demand of wireless and batteryless powering can be caused by different reasons. When the powering wires can not be guided to the sensor (e.g. rotating device, homogeneity of the surface ...), usually the battery powering take place while this is not suitable for every application. Main disadvantage of the battery powering is the limited lifetime and sometimes an improper environment for the battery (e.g. high temperature). In those cases the wireless powering must be applied.

Wireless powering can be performed for instance using the solar cells, temperature difference cells or vibration harvesting. This paper focuses on the energy from the magnetic field which is injected to the system. This solution is proper only for short distances; typically several centimeters. The principle is similar to the RFID but it is more powerful and allows separated powering and communication.

\section{Inductive Coupling}

When two or more inductances partly share the magnetic field, the coupling is presented. The voltage transfer between the coils can be calculated using the Faraday's law. The secondary voltage depends on the secondary coils geometry and on the magnetic field distribution of the primary inductance.

\section{A Coupling Coefficient}

In terms of the circuit theory the inductance coupling can be described using the mutual inductance or using the coupling coefficient. The coupling coefficient is more suitable because the standard simulation tools can be used for a description of the powering. As well as the voltage transfer also the coupling coefficient is given by the coils geometry and the magnetic field distribution. Most important topology of the inductances is the axial orientation. Coefficients dependency versus the distance is given by the magnetic field intensity distribution and is given by the figure 1 . It shows the measurement result of the coupling coefficient between the circular coil and the rectangular surface coil of similar overall dimensions (about $15 \mathrm{~cm}$ ). This dependency is approximated using the exponential and the $1 / \mathrm{x}^{3}$ functions. Character of this dependency is changing approximately at the distance equal to the diameter of the bigger coil (vertical dash line in the picture). This knowledge of the coefficients character can be used for a prediction of the coefficients value for the other coils.

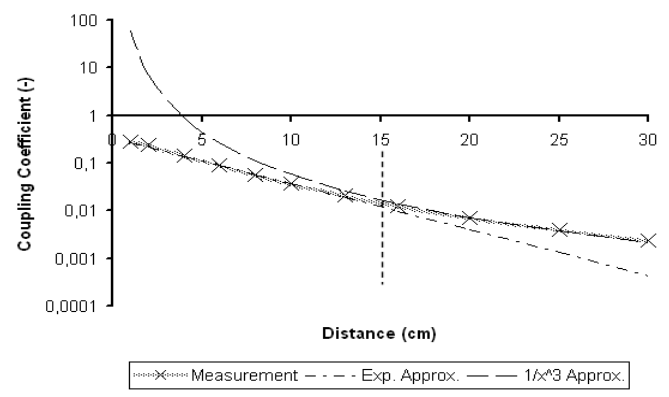

Fig. 1. Approximation by the exponential and $1 / \mathrm{x}^{3}$ functions for the coupled coils

The most important parameters of the coupled inductances are the voltage transfer (1) and the input impedance which can be seen on the inductor $L_{l}$ (2) [1].

$$
\begin{gathered}
\hat{V}_{2}=\hat{V}_{1} \cdot \frac{k \cdot \hat{Z}_{L} \cdot \sqrt{\frac{L_{2}}{L_{1}}}}{\hat{Z}_{L}+j \omega L_{2} \cdot\left(1-k^{2}\right)} \\
\hat{Z}_{\text {in }}=\frac{\hat{V}_{1}}{\hat{I}_{1}}=\frac{j \omega L_{1} \cdot \hat{Z}_{L}+j \omega L_{1} \cdot j \omega L_{2} \cdot\left(1-k^{2}\right)}{\hat{Z}_{L}+j \omega L_{2}}
\end{gathered}
$$


The impedance depends on the loading impedance $Z_{L}$ and on the coupling coefficient $k$.

\section{B Equivalent Circuit}

For an understanding the effects in the circuit it is helpful to derive an equivalent circuit of the coupled inductances. Figure 2 shows the equivalent circuit of one pair of the coupled inductances $L_{1}, L_{2}$ from the figure 3 (e.g. $L_{1}$, $\left.L_{2 \text { pow. }}\right)$. Derivation of this circuit can be realized by observing the circuit behavior under the loading impedance change $[1,4]$.

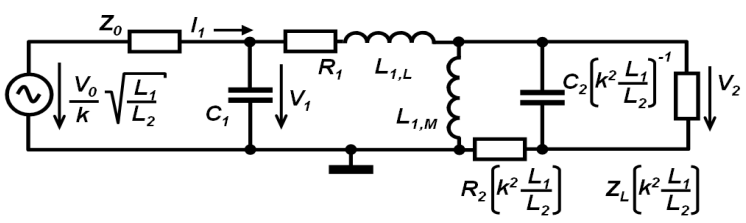

Fig. 2. Equivalent circuit respecting the parasitic properties of the inductances [1]

\section{Resonance Behavior}

It is evident from the Fig. 2, that the circuit exhibits some resonant behavior. Omitting the parasitic effects, the parallel resonance frequency $f_{r p o}$ can be expressed by the (3). The inductance $L_{e}$ is a parallel combination of the $L_{l, L}$ and $L_{l, M}$. Parallel capacitance $C_{p}$ is the transformed secondary side capacitance.

$$
f_{r p 0}=\frac{1}{2 \cdot \pi \sqrt{L_{e} C_{p}}}=\frac{1}{2 \cdot \pi \sqrt{L_{2} \cdot C_{2} \cdot\left(1-k^{2}\right)}}
$$

The voltage transfer in resonance can exceed the nominal transfer (1) (up to over thousands of percents). This effect is crucial part of the powering strategy because the voltage level on the load must be high enough for rectification.

The current flowing to the primary inductance is also depending on the loading impedance. Thus the signal transfer from the sensing probe can be realized using the current measurement in the primary inductance. This is basic principle of the passive RFID systems.

Unfortunately this concept can be realized only for very power-modest circuits because if the secondary side is loaded, the signal transfer is ineffective.

\section{Powering Strategy}

When the power consumption is too big, the simple RFID powering scheme can not be applied. It is important to separate the powering and the communication line.

One possibility is to implement some power management and divide the operation into three phases. In the first phase the circuit is collecting the energy. When there is enough energy the measurement can be performed and the last phase is the signal transfer. This concept is sometimes useful but has disadvantage that the measurement can not be continual and sometimes the capacitor for energy storage must be too big.

Other possibility is to divide the powering and communication by the frequencies bands. Figure 3 shows modified RFID principle, where the powering and communication is has its own receiving inductance and the frequencies can be selected to be different.

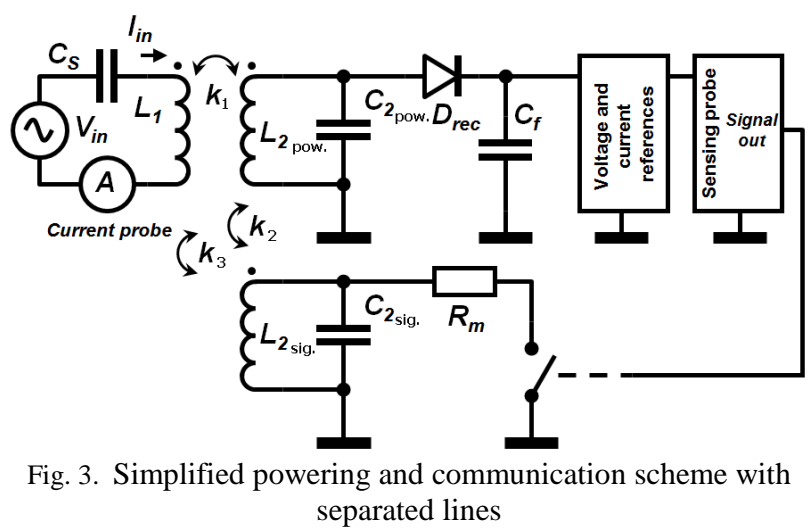

The inductance $L_{2 p o w}$ is powering the electronics and the inductance $L_{2 s i g}$ serves for the signal transfer. The coupling $k_{2}$ between the $L_{2 p o w}, L_{2 s i g}$ is parasitic and should be minimal in order to keep the lines separated. Signal transfer from the sensing probe is sensed using the current probe on the inductance $L_{l}$. For each position of the switch it is different also the input current at given frequency because the modulating resistor $R_{m}$ changes the quality factor. Loading impedance represented by the sensing probe is stable and on different frequency. Current change on the input is thus caused mainly by the switch.

\section{Receiving inductances}

The goal of this paper is to maximize the powering ability and keep the possibility of passive signal transfer. The crucial devices for this are the secondary inductances. Figure 4 presents two possible configurations.
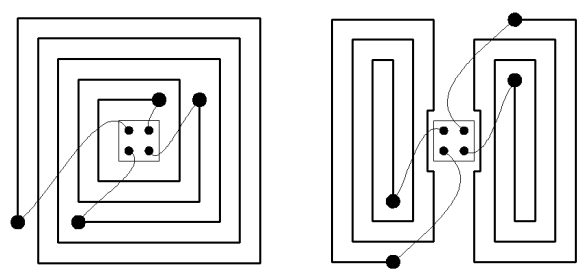

Fig. 4. Two possible configurations of powering and signal inductances, powered device is in the center

The overall geometry of the antennas is given by demand of application. It is presumed the surface mounting, thus the rectangular surface antennas were tested. In the center there is situated the sensor and the electronics. Two basic concepts of location were tested whereat the total surface of the antennas is equal. Power and signal-transfer ability for both configurations are presented on the figures 5 and figure 6 . 

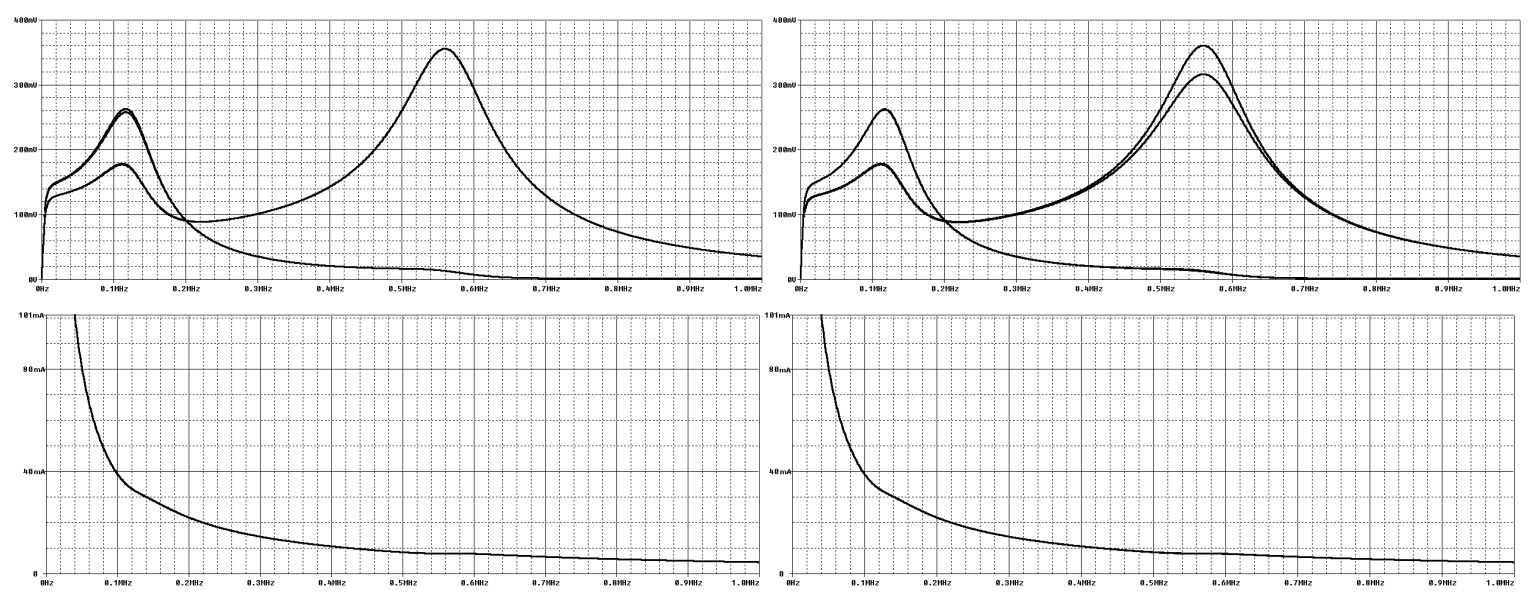

Fig. 5. Simulation results for concentric configuration of the antennas (according the figure 4, left)

\section{Simulation results}

\section{A Properties of the antennas}

Presented results were simulated according the figure 3. The transmitting antenna $L_{l}$ is always simple winded circular coil of winding number 10 , diameter $15 \mathrm{~cm}$ and inductance $39 \mu \mathrm{H}$. Coupling coefficients $k_{1}$ and $k_{3}$ were measured and it is 0.25 .

Receiving antennas $L_{2 p o w}, L_{2 s i g}$ are squared and were performed on the cuprextit board. They are of different dimension for different configurations to keep the total surface equal.

The axial configuration of the antennas has following properties. The powering inductance has winding number 8 , diameter $10 \mathrm{~cm}$, inductance $14 \mu \mathrm{H}$. Signal inductance has winding number 8 , diameter $8 \mathrm{~cm}$, inductance $10.6 \mu \mathrm{H}$. Coupling $k_{2}$ between the inductances was measured to be 0.44 .

The configuration side-by-side has different parameters for the simulation. The powering inductance and the signal inductance are same, it has winding number 21 , diameter $9 \times 4.5 \mathrm{~cm}$, inductance $21 \mu \mathrm{H}$. Coupling between the inductances was measured to be 0.024 .
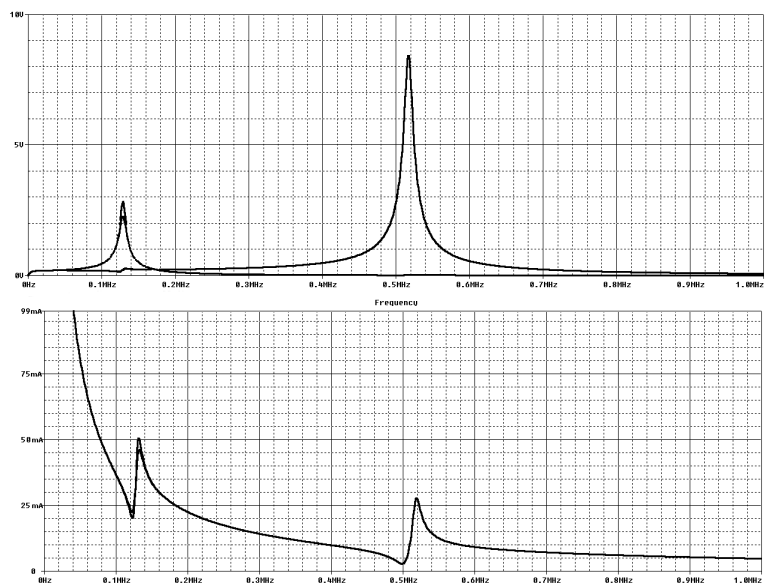

\section{B Estimation of the results}

The figures 5 and 6 show the AC analyses of the voltages and currents on the antennas. It is for the input voltage $1 \mathrm{~V}$ and different loading resistance $(1 \mathrm{k} \Omega, 10 \mathrm{M} \Omega)$ on the signal antenna (simulation of the modulating switch). The load on the powering antenna is stable $10 \mathrm{k} \Omega$. The pictures present voltage on the powering antenna (up) and current of the transmitting antenna $\mathrm{L}_{1}$ (down). The left figures are for the modulation switch on the lower frequency antenna and the right figures are for the high frequency modulation. Crucial features to be estimated are the stability of the powering voltage, good response of the input current on the loading impedance change.

Because of big coupling coefficient between the receiving antennas for the axial configuration the modulating switch is affecting the powering line and the powering thus stops to be effective. Also the current change is negligible in this configuration.

For side-by-side configuration the performance of the powering is stable and nearly independent on the situation on the signal line. This configuration must be preferred in order to maximize the modulation depth. This ensures possibility of sensing the signal for long distances and simultaneously provides enough energy for powering

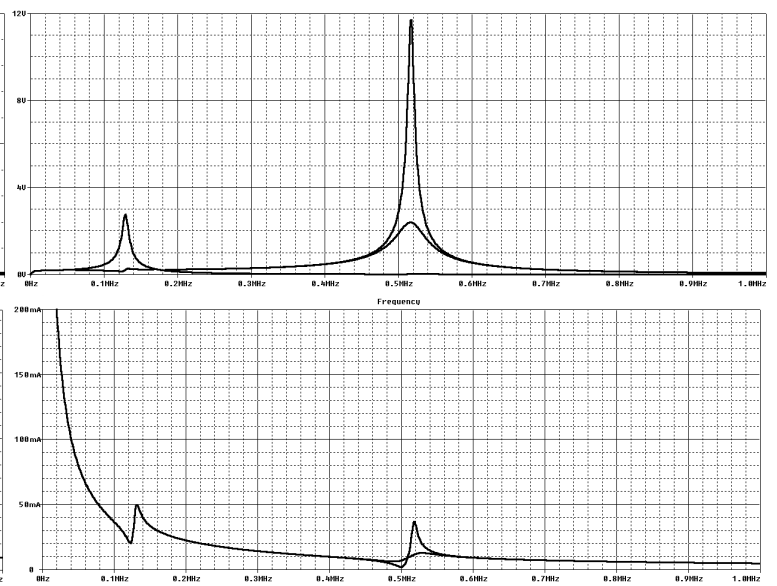

Fig. 6. Simulation results for configuration of the antennas side by side (according the figure 4, right) 


\section{Circuit realization}

Circuit from the figure 3 was realized using the discrete devices and tested with the side by side configuration of the antennas. As the sensor for measurement it was tested the accelerometer ADXL 203 and strain gauges [2].

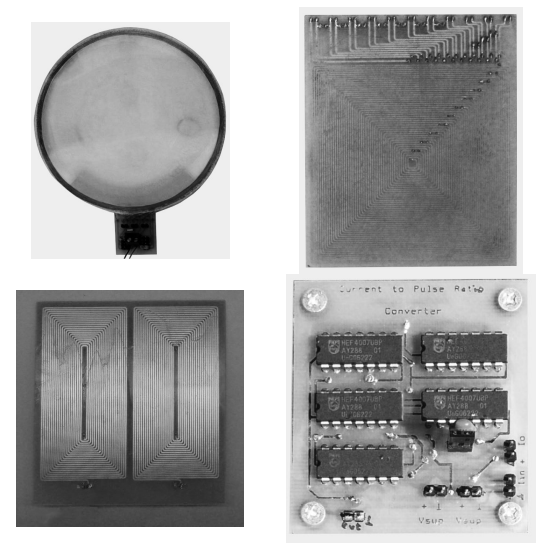

Fig. 7. Realization of the sensing probe using the discrete devices (right, down) and transmitting and receiving antennas

The measurement was effective up to distances of $10 \mathrm{~cm}$. Accuracy of the measurement was limited by the simplicity of the realized converter and it was about $15 \%$. Nowadays the integrated circuit is prepared. It will be finished in March 2011. The expected performance is $15 \mathrm{~cm}$ distance ability and the accuracy better then $5 \%$.

\section{Conclusion}

The paper presented wireless powering solution for measurement in the isolated areas which can be used for long-term monitoring of contamination of physical damage in barrels, concrete walls etc.

It is provided by the near magnetic field and the signal transfer principle is similar to the RFID systems. There are presented possibilities of maximizing the power transfer in order to perform high power measurement (high relatively to the RFID systems). The paper focused on dual frequency solution - one frequency for powering other for the signal transfer. There are two possible configurations of the antennas. The side-by-side configuration appears to be the best solution.

Functionality of the system was tested on the circuit consisting of discrete devices and which is able to measure with the accelerometer ADXL203 and the strain gauges. The measurement was effective up to distances of $10 \mathrm{~cm}$. Nowadays the integrated circuit is prepared. It will be finished in March 2011. The expected performance is $15 \mathrm{~cm}$ distance ability and the accuracy better then $5 \%$.

As the transmitting antenna $\left(L_{l}\right)$ was used simple-winded circular coil and the receiving antennas were performed on the cuprextit board and attached to the circuit.

\section{Acknowledgement}

Research described in the paper has been supported by the Czech Science Foundation project No. 102/09/1601 "Micro- and nano-sensor structures and systems with embedded intelligence" and partially by the research program No. MSM 6840770012 "Transdisciplinary Research in Biomedical Engineering II" of the CTU in Prague.

\section{References}

[1] A. Boura, M. Husak: "Powering for Long Term Monitoring in the Enclosed Areas", in Proc. ICREPQ'10, Granada (Spain), March 2010, \#414

[2] P. Kulha, A. Boura, M. Husak: "Design and Fabrication of Piezoresistive Strain-Gauges for Harsh Environment Applications", in Proc. ICREPQ'10, Granada (Spain), March 2010, \#510

[3] T. Prochazka, "Antennas for RFID systems", Elektrorevue, 2002, vol. 22, available at http://www.elektrorevue.cz/ clanky/02022/index.html, 2009.

[4] Wu, J., Quinn, V., Bernstein, G. "A simple, wireless powering scheme for MEMS devices", MEMS Components and Applications for Industry, Automobiles Aerospace, and Communications, Proceedings of SPIE, 2001, Vol. 4559, URL: http://www.ece.utk.edu/ jaynewu/Papers/SPIE\%202 001\%204559_7.pdf 